\title{
Impact de la liquidation d'une option sur un marché d'action
}

\section{Cécile BOYER ${ }^{1}$, Gabrielle DEMANGE ${ }^{2,3}$}

RÉSUMÉ. - Nous montrons dans cet article que la liquidation d'une option offre des opportunités de manipulation importantes du cours de l'action sous-jacente. Dans une enchère en fonctions de demande, les positions en option des investisseurs stratégiques affectent fortement les stratégies d'équilibre. La convexité du paiement de l'option justifie l'utilisation de fonctions de demandes croissantes qui entraîne des discontinuités sur le prix d'échange. De plus l'option induit des complémentarités stratégiques entre ses détenteurs, ce qui génère des équilibres multiples, même dans la classe des équilibres dits linéaires.

\section{Imperfect competition on stock markets: the impact of an option at the exercice date}

ABSTRACT. - We examine whether the liquidation of an option offers new opportunities of price manipulation on the underlying stock. In a call auction on the stock at the option exercise date, traders compete through demand schedules. The convexity of the option payoffs may induce equilibrium demand schedules to be increasing in price, which rationalize stop orders and generate discontinuities in the exchange price. Moreover, multiple equilibria may exist even in the class of linear equilibria, due to positive externalities among option holders.

\footnotetext{
1 THEMA, CREST, adresse : THEMA - Université de Cergy-Pontoise, 33 boulevard du Port, 95011 Cergy-Pontoise Cedex, tel. 013425 61 80, fax. 01342562 33, E-mail boyer@ensae.fr

2 EHESS-DELTA, CEPR, adresse : DELTA - ENS, 48 bd Jourdan, 75014 Paris, tel. 01431363 27, fax. 01431363 10, E-mail demange@ delta.ens.fr

3 Nous remercions le rapporteur et P. ROGER pour leurs commentaires. Gabrielle DEMANGE remercie aussi le groupe de finance de l'ESCT.
} 


\section{Introduction}

L'évolution des marchés financiers a été marquée durant les vingt dernières années par l'essor des produits dérivés tels que les options américaines ou européennes, les swaps, les contrats à terme... La théorie économique permet d'expliquer ce phénomène. En complétant les marchés, les actifs dérivés offrent aux investisseurs de nouvelles possibilités de partage du risque (Ross [1976], Demange-LaroQue [1999]). Ce résultat repose toutefois sur l'hypothèse, simplificatrice mais peut-être injustifiée, du comportement de " preneur de prix » (price taker) des investisseurs. Depuis longtemps, les détenteurs de contrats à terme ont pu tirer profit d'un rationnement du sous-jacent, par le biais de manœuvres de "squeeze » ou de " corner », lorsque la livraison physique du sous-jacent est exigible ${ }^{4}$. Si le règlement du contrat s'effectue par simple compensation monétaire (le cas le plus répandu pour les contrats dérivés sur produits financiers), des investisseurs stratégiques peuvent aussi être incités à manipuler le prix du sous-jacent puisque celui-ci affecte le paiement du contrat dérivé. Notre objectif est d'étudier ce cas lorsque le titre dérivé est une option européenne ou encore un bon de souscription en actions ${ }^{5}$. Rappelons qu'une option d'achat européenne peut être exercée par son détenteur à un prix et à une date déterminés (appelés prix et date d'exercice). Alors que le paiement d'un contrat à terme est linéaire dans le prix du sous-jacent, celui d'une option est convexe grâce à la faculté de ne pas exercer l'option si le cours de l'action est inférieur au prix d'exercice. Nous montrerons que cette convexité affecte fortement le comportement d'un investisseur stratégique en fonction de sa position sur l'option.

L'analyse est conduite à la date de liquidation lorsque l'option n'est plus échangée et que le prix du sous-jacent détermine le règlement. Dans ces conditions, un investisseur en position longue sur l'option sera tenté d'augmenter sa demande d'actions (par rapport à la situation où il ne détiendrait pas d'option) afin d'obtenir une hausse des cours, augmentant ainsi à la fois la probabilité d'exercice de l'option et son paiement en cas d'exercice. Toutefois, une telle manœuvre n'est bénéfique que si le gain sur l'option excède la perte sur l'action (liée à l'accroissement joint du prix et de la demande). Inversement, un investisseur en position courte sur l'option envisagera d'augmenter son offre d'actions pour favoriser une baisse des cours. Le bien-fondé de telles stratégies doit être examiné dans un cadre d'équilibre.

\footnotetext{
4. La livraison, bien que plus courante pour les contrats sur marchandises, est aussi exigée par certains contrats sur produits financiers. L'exemple le plus connu est sans doute le contrat à terme sur les Bons nouvellement émis par le Trésor américain. Il pourrait expliquer les manœuvres de squeeze dont SALOMON BROTHERS a été accusé, et qui ont conduit à une modification des règles d'enchères sur les émissions du marché primaire [voir BIKCHANDANI-HuANG (1993) et JEGADEESH (1993)].

5. Les caractéristiques des options et bons de souscription en actions sont similaires. Cependant, alors que les options, organisées par une bourse, sont détenues en somme nulle par les investisseurs, les bons de souscription ou « stock options », émises par l'entreprise, sont détenues en somme positive.
} 
Dans cet article, nous considérons le marché de l'action, lorsque les investisseurs s'y livrent une concurrence en fonction de demande. Une fois les demandes soumises, un prix d'échange est sélectionné, si possible afin d'équilibrer le marché ${ }^{6}$. Un tel mécanisme est à rapprocher d'un fixing tel que celui de la Bourse de Paris où toute fonction de demande peut être approchée par une combinaison des ordres autorisés. Dans ce cadre, l'utilisation d'ordres stop, c'est-à-dire d'ordres d'achat (resp. de vente) qui ne sont exécutés que si le prix augmente (resp. diminue) suffisamment, permet d'obtenir des demandes localement croissantes.

Deux types d'investisseurs interviennent sur le marché de l'action : d'une part, des investisseurs stratégiques au pouvoir de marché important, de l'autre une multitude de petits investisseurs concurrentiels. Tous sont rationnels, présentent de l'aversion pour le risque et peuvent détenir des parts du dérivé. Les investisseurs stratégiques représentent des institutions financières (banques, fonds de placement ou « hedge funds ») qui interviennent régulièrement sur le marché. Les investisseurs concurrentiels au contraire ont une participation moins bien identifiée, aussi leur demande est soumise à un aléa non observé.

Afin de dégager les spécificités induites par la non-linéarité du paiement de l'option, nous étudions tout d'abord le cas où les investisseurs détiennent des contrats à terme (futures) à la place des options. L'équilibre est alors unique ; de plus les fonctions de demande sont linéaires, et le prix d'échange est continu par rapport à l'aléa sur la demande concurrentielle (en fait il en dépend même de façon linéaire). Ce résultat ne se généralise pas à un contrat optionnel qui, du fait de la convexité de son paiement, introduit deux effets principaux. D'une part, les prix d'échange peuvent être discontinus par rapport aux aléas, lorsque les détenteurs d'option trouvent profitable de soumettre une demande localement croissante afin d'éloigner le prix d'échange du prix d'exercice. Nous justifions ainsi l'utilisation d'ordres stop (qui permettent d'obtenir des demandes croissantes) par des raisons stratégiques. Ceci est à contraster avec GENOTTE et LELAND [1990] qui, en supposant a priori l'utilisation d'ordres stop, ont montré que la nonmonotonie des fonctions de demande pouvait générer des discontinuités dans les prix. D'autre part, l'option induit des complémentarités stratégiques entre les investisseurs qui l'ont acheté. La multiplicité des équilibres qui peut en résulter fragilise la réalisation d'un équilibre : comment les investisseurs se coordonnent-ils sur un équilibre plutôt que sur un autre ?

$\mathrm{Au}$ vu de ces résultats, la liquidation de l'option semble avoir un effet déstabilisant sur le cours de l'action sous-jacente. L'effet stabilisant ou non des marchés à terme a été abondamment étudié (voir par exemple DANTHINE [1978], Turnovski [1983], JARrow [1992]) dans le cas de la livraison physique du sous-jacent, KUMAR-SEPPI [1992] dans le cas de règlements monétaires, STEIN [1987] et BACK [1993] en asymétrie d'information). À notre connaissance cet article est le premier à montrer qu'une option, du fait de la convexité de son paiement, induit un nouvel élément déstabilisant.

6. Un mode de concurrence similaire est étudié dans le cadre des enchères de biens divisibles par WILSON [1989], d'un duopole servant une demande aléatoire par KLEMPERER-MEYER [1989] ou encore d'un marché d'action en présence d'initiés par KYLE [1989] ou BHATTACHARYA-SPIEGEL [1991]. 
Le modèle est décrit dans la partie suivante. Après l'étude du comportement d'un individu stratégique (partie 3), nous étudions les équilibres avec un ou plusieurs investisseurs stratégiques (parties 4 et 5). La conclusion et les preuves figurent dans les deux dernières parties.

\section{Le modèle}

Nous analysons le marché d'une action à une seule date, $t=0$, qui coïncide avec la date de liquidation d'une option d'achat européenne sur cette action. L'option n'est plus échangée et le prix de l'action à cette date détermine le paiement de l'option. Le prix d'exercice de l'option est $K$. La détention d'une unité de l'option ${ }^{7}$ rapporte le montant $(p-K)^{+}=\operatorname{Max}(0, p-K)$, si le prix d'échange de l'action est $p$.

Ce prix est fixé par le mécanisme suivant. Tous les investisseurs soumettent au marché une fonction de demande contingente au prix par le biais d'un commissaire-priseur ou d'un système automatique. Ce dernier sélectionne ensuite un prix d'échange qui annule la demande excédentaire (ou, si cela est impossible, qui la minimise). Les investisseurs reçoivent les quantités qu'ils ont demandées à ce prix.

Une telle procédure est à rapprocher des marchés réels de fixing dirigés par les ordres au sein desquels les investisseurs soumettent différents types d'ordres : ordre au mieux (c'est-à-dire une quantité valable pour tous les prix, assimilable à une fonction de demande constante), ordre limite (une quantité et un prix maximum dans le cas d'un ordre d'achat ou un prix minimum dans le cas d'un ordre de vente) ou ordre à seuils de déclenchement, encore appelé ordre stop (une quantité et le prix minimum à partir duquel l'investisseur l'achète ou le prix maximum à partir duquel il la vend). Toute fonction de demande peut être approchée par une combinaison de tels ordres. Une croissance en les prix de la fonction de demande traduit l'utilisation d'ordres stop. Notons que le prix d'échange et donc le paiement de l'option sont affectés par chacune des demandes. Ceci suggère que les positions sur l'option influent sur les comportements stratégiques.

Deux types d'investisseurs interviennent sur le marché. Les premiers, non concurrentiels ou stratégiques, connaissent l'influence de leur demande sur le prix d'échange et tentent d'en tirer profit. Les investisseurs concurrentiels sont plus nombreux et de petite taille. Ils ne peuvent soumettre des demandes suffisamment importantes pour influencer le prix de facon significative et de ce fait adoptent un comportement concurrentiel : ils ne tiennent pas compte de l'influence de leur demande sur le prix.

Les investisseurs stratégiques et concurrentiels sont indicés respectivement par les lettres $i \in I, I=\{1, \ldots, n\}$ et $j \in J, J=\{1, \ldots, m\}$. Ils échangent deux actifs : l'action (risquée) et un actif sans risque de rendement normalisé à 1 , la 7. Notre analyse se transpose aisément à une option de vente qui procure
$(K-p)^{+}=\operatorname{Max}(0, K-p)$. 
monnaie. Une unité d'action vaudra ${ }^{8} \tilde{v}$, appelée valeur de l'action, à une date future $t=1$. Tous les agents ont des préférences représentées par des fonctions d'utilité de VON NEUMANN-MORGENSTERn, de coefficient d'aversion absolue pour le risque constant $\rho_{k}: U_{k}(w)=-\exp \left(-\rho_{k} w\right)$. L'investisseur $k$, $k=i, j$, détient initialement $a_{k}$ unités d'option et $b_{k}$ unités d'action ${ }^{9}$. Si l'agent $k$ achète $x$ unités d'action au prix $p$, sa richesse à la fin du fixing vaut

$$
x(\tilde{v}-\tilde{p})+a_{k}(\tilde{p}-K)^{+}+b_{k} \tilde{v} .
$$

Comme on l'a dit, les investisseurs soumettent au marché des fonctions de demandes contingentes au prix, notées $d_{k}(),. k=i$ ou $j$.

\section{Le prix d'échange}

Une fois les demandes $d_{k}, k=i, j$, soumises, la procédure de fixing sélectionne un prix d'échange qui minimise (en valeur absolue) l'excès de demande. Dans la suite, il existera toujours un unique prix $p^{*}$ annulant la demande excédentaire, c'est-à-dire vérifiant :

$$
\sum_{k \in I \cup J} d_{k}(p)=0 .
$$

Il sera donc retenu comme prix d'échange. L'investisseur $k$ reçoit alors la quantité $d_{k}\left(p^{*}\right)$. Notons que toutes les unités d'action sont achetées ou vendues au même prix : le procédé se rapproche d'une enchère uniforme.

\section{Les hypothèses}

1. Le portefeuille de l'investisseur stratégique $i$, caractérisé par $\left(a_{i}, b_{i}\right)$, est connu de tous. Nous notons $a_{I}=\sum_{i \in I} a_{i}$ et $b_{I}=\sum_{i \in I} b_{i}$. Les portefeuilles des investisseurs concurrentiels $j$ sont des informations privées. La somme de leurs dotations initiales en action, $\tilde{b}_{J}=\sum_{j \in J} \tilde{b}_{j}$, est en particulier perçue comme une variable aléatoire.

2. La valeur fondamentale $\tilde{v}$ est normalement distribuée, d'espérance $\bar{v}$ et d'écart-type $\sigma_{v}$. Elle est indépendante des portefeuilles des agents concurrentiels $\left(\tilde{a}_{j}, \tilde{b}_{j}\right), j \in J$. Aucun investisseur ne détient d'information privée sur $\tilde{v}$.

3. Les investisseurs stratégiques présentent une aversion stricte pour le risque, de même coefficient $\rho>0$. Nous notons $\rho_{I}$ leur coefficient agrégé : $\rho_{I}=\rho / n$. De même le coefficient agrégé d'aversion absolue pour le risque des investisseurs concurrentiels est supposé positif, noté $\rho_{J}$.

8. Dans la suite, un sera apposé au dessus de toutes les variables aléatoires ; leurs réalisations seront notées sans .

9. Il est inutile de spécifier les dotations monétaires des investisseurs qui n'influent pas les demandes d'actions sous l'hypothèse d'aversion absolue constante. Notre analyse s'étend facilement au cas où les dotations initiales sont des portefeuilles d'actifs plus diversifiés, sous l'hypothèse de normalité des rendements ; $b_{i} \tilde{v}$ représente alors la part du rendement du portefeuille corrélée à $\tilde{v}$ et la partie indépendante n'a aucun impact sur les décisions de l'agent qui portent sur l'action. 
Si l'on assimile les investisseurs non concurrentiels à des institutions financières, présentes de façon régulière sur le marché, supposer leurs positions connues (hypothèse 1) ne parait pas déraisonnable (cette hypothèse peut d'ailleurs être en partie relâchée sans changer fondamentalement les résultats mais au prix de difficultés techniques supplémentaires qui alourdiraient l'exposé). À l'inverse, il est naturel que le portefeuille des petits investisseurs soit imparfaitement connu. De ce fait, leur demande est aussi imparfaitement connue, ce qui induit un aléa dans le prix d'échange.

On pourrait aussi examiner le jeu dans lequel les investisseurs choisissent leur portefeuille dès l'émission de l'option, tout en anticipant la manipulation du prix du sous-jacent lors de la liquidation ${ }^{10}$. L'analyse d'un tel jeu n'est pas aisée dans un cadre réaliste où l'espérance de la valeur fondamentale varie avec le temps. De plus elle n'est pas pertinente si, comme on peut le penser, la motivation première d'une institution pour acheter ou vendre une option bien avant sa date d'exercice est la gestion de son portefeuille et non pas la perspective de manipulation du prix du sous-jacent à l'exercice ${ }^{11}$. Par contre si une opportunité de manipulation se présente à l'échéance de l'option, elle est vraisemblablement exploitée.

Rappelons que les positions agrégées des investisseurs sont nulles sur une option échangée sur un marché organisé et sont positives sur une option émise par une entreprise (bon de souscription en actions ou stock option). Cependant, même dans le premier cas, du fait des investisseurs concurrentiels, la somme des positions sur l'option des investisseurs stratégiques $\sum_{i \in I} a_{i}$ n'a pas de raison d'être nulle. Nous considérerons aussi le cas où les positions des investisseurs stratégiques sont toutes positives sur l'option, ce qui est justifié pour le second type d'option.

Enfin, nous ne formulons pas d'hypothèse sur les positions relatives du prix d'exercice $K$ et de l'espérance de la valeur fondamentale $\bar{v}$ à l'exercice. Si, à l'émission de l'option, le prix d'exercice est souvent proche du prix courant de l'action, lui-même relié à l'espérance de la valeur fondamentale, cette espérance a pu évoluer dans le temps.

\section{La demande concurrentielle}

Les investisseurs concurrentiels ne prennent pas en compte l'influence de leur propre demande sur le prix d'équilibre ; ils demandent donc pour chaque prix la quantité qui maximise leur utilité espérée. En conséquence, sous les hypothèses citées précédemment, la demande d'un agent concurrentiel est indépendante du montant d'option qu'il détient. Par un argument standard ${ }^{12}$ la demande agrégée des investisseurs concurrentiels est donnée par :

10. Une telle analyse est conduite pour des contrats à terme par KUMAR-SEPPI [1992] avec des investisseurs neutres vis-à-vis du risque.

11. Par exemple une banque vendant des options d'achat au détail à ses clients se couvrira en achetant ces options sur le marché organisé. En effet, les marchés organisés d'option tels que le MONEP ou le MATIF sont difficilement accessibles aux particuliers du fait des montants des contrats. De même, lors de la vente de produits dit structurés, offrant des garanties «planchers », l'institution achètera des options de vente.

12. Du fait des hypothèses de normalité, l'agent $j$ choisit $z_{j}$ de façon à maximiser $z_{j}(\bar{v}-p)-\frac{\rho_{j}}{2}\left(z_{j}+b_{j}\right)^{2} \tau_{v}^{2}$. Sa demande individuelle vaut donc $z_{j}\left(p, b_{j}\right)=\frac{\bar{v}-p}{\rho_{j} \sigma_{v}^{2}}-\tilde{b}_{j}$ 


$$
\sum_{j \in J} d_{j}(p)=\frac{\bar{v}-p}{\rho_{J} \sigma_{v}^{2}}-b_{J}
$$

Elle s'interprète comme la somme d'une demande spéculative, sensible à l'écart du prix à l'espérance de la valeur, et d'une demande de couverture dont le but est d'éliminer le risque du portefeuille final. Aussi par la suite appelons-nous $\tilde{b}_{J}$ l'offre de couverture.

Si tous les agents étaient concurrentiels $(I=\emptyset)$, le prix d'échange serait donné par :

$$
p=\bar{v}-\rho_{J} \sigma_{v}^{2} b_{J} .
$$

Il serait ainsi indépendant de la distribution des options entre les investisseurs et continu, en fait même linéaire, par rapport aux aléas de l'offre de couverture.

\section{Équilibre}

Un agent stratégique choisit la fonction de demande qui maximise son utilité espérée en prenant en considération l'influence sur le prix d'échange de son propre investissement en action. Comme il sait que le prix d'échange est donné par (1), cette influence est appréhendée à travers ses anticipations quant aux demandes des autres investisseurs. Nous supposerons ses anticipations correctes. Si un seul investisseur stratégique est présent sur le marché, cela signifie simplement qu'il sait que la demande concurrentielle est donnée par (2). Si plusieurs investisseurs sont stratégiques, chacun d'entre eux sait de plus comment sont formées les demandes des autres investisseurs stratégiques et optimise en conséquence son utilité espérée. Autrement dit, nous considérons un équilibre NASH-BAYÉSIEN entre les investisseurs non concurrentiels, dans lequel chaque stratégie est une meilleure réponse aux autres stratégies.

DÉFINITION 1 Les fonctions de demande $\left(d_{i}(),. i \in I\right)$ forment un équilibre si, pour tout $i \in I, d_{i}($.$) maximise$

$$
E\left[U_{i}\left(x(\tilde{p})(\tilde{v}-\tilde{p})+a_{i}(\tilde{p}-K)^{+}+b_{i} \tilde{v}\right)\right]
$$

sur l'ensemble des fonctions de demande $x($.$) , sachant que le prix est donné$ par

$$
x(\tilde{p})=-\sum_{k \neq i, k \in I} d_{k}(\tilde{p})+\frac{p-\bar{v}}{\rho_{J} \sigma_{v}^{2}}+\tilde{b}_{J} .
$$

Avant d'étudier les équilibres il n'est pas inutile de mentionner l'impact de l'aléa sur l'offre de couverture $\tilde{b}_{J}$. En l'absence d'un tel aléa, en un équilibre, chaque investisseur connaît parfaitement la fonction d'offre à laquelle il est confronté, et donc le prix qui résultera de sa demande. De par la flexibilité apportée par une fonction de demande, il existe alors une infinité d'équilibres de NASH (voir par exemple KLEMPERER et MEYer [1989] dans le cadre d'un 
duopole). Dans chaque équilibre, s'opère une coordination implicite sur un prix donné ; elle est stabilisée car les demandes pour les autres prix spécifient des «punitions » en cas de déviation. En présence d'aléas sur la courbe d'offre, le prix d'équilibre est aléatoire, et de telles punitions sont trop coûteuses : la manipulation est plus difficile.

Nous analysons d'abord le comportement optimal d'un investisseur vis-àvis des stratégies des autres investisseurs.

\section{Meilleure réponse face à une fonc- tion d'offre aléatoire}

Remarquons d'abord que seule l'offre agrégée des autres intervenants, concurrentiels ou non, importe pour un investisseur (d'après l'équation (3)). Pour déterminer la meilleure réponse d'un investisseur face à une courbe d'offre aléatoire, nous nous appuierons sur l'idée suivante : conditionner sa demande par rapport au prix est équivalent à la conditionner par rapport à un aléa inobservé dès lors que le prix d'échange est une fonction injective de l'aléa. Plus précisément, soit $O$ l'offre à laquelle l'investisseur fait face, offre qui dépend non seulement du prix mais aussi du paramètre aléatoire et non observable $\tilde{\varepsilon}: O(p, \tilde{\varepsilon})$. Si l'investisseur observe $\varepsilon$, il peut choisir n'importe quel couple quantité-prix $(x, p)$ sur la courbe d'offre $x=O(p, \varepsilon)$. Supposons sa transaction préférée unique et notons-là $(O(p(\varepsilon), \varepsilon), p(\varepsilon))$. Si la fonction $\varepsilon \rightarrow p(\varepsilon)$ est injective, l'investisseur peut soumettre la fonction de demande $p \rightarrow d(p)=O(p, \varepsilon(p))$ où $\varepsilon(p)$ est la fonction réciproque de $p($.$) . Il obtient$ alors pour toute réalisation $\varepsilon$ sa transaction préférée sur la courbe d'offre comme s'il connaissait $\varepsilon$ : cette demande constitue sûrement une meilleure réponse à l'offre aléatoire considérée.

Ce principe, utilisé entre autres dans KYLE [1989] et KLEMPERER-MEYER [1989], guidera certaines démonstrations même si l'hypothèse d'injectivité n'est pas toujours satisfaite dans notre modèle. Il s'applique directement si l'option est remplacée par un contrat à terme. Rappelons qu'un contrat à terme sur l'action de mêmes prix et date d'exercice que l'option procure le gain $p-K$ à la date de liquidation. Les paiements de l'option et du contrat à terme sont donc identiques quand l'option est exercée. Aussi la proposition suivante qui détermine la meilleure réponse d'un investisseur détenant un contrat à terme nous sera-t-elle utile par la suite. De plus, elle nous permettra, par comparaison, de mieux comprendre les conséquences de la convexité du paiement de l'option.

Proposition 1 Soit un investisseur détenant le portefeuille $(a, b)$ de contrat à terme et d'action sous-jacente. S'il fait face à la courbe d'offre définie par

$$
o_{\gamma}(p)+\tilde{\varepsilon}=\frac{p-\bar{v}}{\gamma \sigma_{v}^{2}}+\tilde{\varepsilon}
$$


où $\gamma$ est un réel positif et $\tilde{\varepsilon}$ une variable aléatoire, la fonction de demande suivante constitue une meilleure réponse :

$$
d_{\gamma, a, b}(p)=\frac{\bar{v}-p}{(\gamma+\rho) \sigma_{v}^{2}}+\frac{\gamma a-b \rho}{\gamma+\rho} .
$$

Le prix d'échange est alors donné par :

$$
p_{\gamma, a, b}(\tilde{\varepsilon})=\bar{v}-\left(\frac{1}{\gamma+\rho}+\frac{1}{\gamma}\right)^{-1}\left(\frac{\rho b-\gamma a}{\gamma+\rho}+\tilde{\varepsilon}\right) \sigma_{v}^{2}
$$

Si l'investisseur pense qu'il n'a aucune influence sur le prix $(\gamma=0)$, il soumet sa demande concurrentielle. Conformément à l'intuition, la distorsion par rapport à cette demande concurrentielle est d'autant plus grande qu'il peut influer sur le prix, c'est-à-dire que $\gamma$ est élevé.

\section{4 Équilibre avec un risque investis- seur stratégique}

Dans cette partie, nous supposons qu'un seul intervenant adopte un comportement non concurrentiel. De ce fait nous l'appelons monopole et notons $a$ et $b$ ses positions en omettant l'indice $i$. Ce monopole fait face à l'offre linéaire concurrentielle (nous notons $o_{J}$ la fonction $o_{\rho_{J}}$ afin de simplifier les notations) :

$$
o_{J}(p)+\tilde{b}_{J}=\frac{p-\bar{v}}{\rho_{J} \sigma_{v}^{2}}+\tilde{b}_{J}
$$

dans laquelle l'offre de couverture $\tilde{b}_{J}$ est inconnue. Supposons dans un premier temps que le monopole puisse observer l'offre de couverture $b_{J}$. Si sa position est longue sur l'option, ses transactions préférées sur les courbes d'offre associées à $b_{J}$ sont discontinues. Plus précisément, il existe un seuil, noté $b^{*}$, pour lequel le prix préféré passe de $\underline{p}$ à $\bar{p}$ avec $\underline{p}<K<\bar{p}$. Cette discontinuité résulte de la convexité du paiement de l'option. En effet, l'investisseur préférera toujours au prix d'exercice $K$ un prix légèrement supérieur afin d'exercer l'option : le gain marginal en $K$ présente un saut positif lié au paiement sur les unités d'option. L'argument est valable pour les prix proches du prix d'exercice qui ne seront donc jamais choisis. Si le monopole détient une position courte sur l'option, il choisira au contraire un prix d'échange égal au prix d'exercice pour de nombreuses valeurs de $b_{J}$, afin d'éviter la perte liée à l'exercice de l'option.

En fait l'investisseur peut presque sûrement obtenir les mêmes transactions s'il ne connaît pas la demande de couverture $b_{J}$, ce qui donne la proposition 2 . 
Proposition 2 Supposons la distribution de $\tilde{b}_{J}$ continue et considérons un investisseur stratégique confronté à la courbe d'offre linéaire et aléatoire $o_{J}(p)+\tilde{b}_{J}$.

Si la position de l'investisseur sur l'option est longue $(a>0)$, il existe une valeur $b^{*}$, des prix $p, \bar{p}$ avec $\underline{p}<K<\bar{p}$ tels que la demande continue, linéaire par morceaux définie par :

$$
\begin{gathered}
d_{\rho_{J}, 0, b}(p) \text { pour } p<\underline{p}, d_{\rho_{J}, a, b}(p) \text { pour } p>\bar{p} \\
\text { et à la fonction d'offre o } o_{J}(p)+b^{*} \text { pour } \underline{p} \leqslant p \leqslant \bar{p} .
\end{gathered}
$$

soit optimale. Les prix entre $\underline{p}$ et $\bar{p}$ ne sont jamais atteints à l'équilibre. Si sa position sur l'option est courte $(a<0)$, une demande optimale est donnée par :

$$
\begin{array}{r}
d_{\rho_{J}, 0, b}(p) \text { pour } p<K, d_{\rho_{J}, a, b}(p) \text { pour } p>K \\
\text { et à l'intervalle }\left[d_{\rho_{J}, a, b}(K), d_{\rho_{J}, 0, b}(K)\right] \text { pour } p=K .
\end{array}
$$

Le prix d'exercice $K$ coüncide alors avec le prix d'échange pour un intervalle de valeurs de $b_{J}$.

La demande du monopole est composée de deux parties. Pour les prix faibles, elle coïncide avec la demande qu'il aurait soumise s'il ne détenait pas d'option, et pour les prix élevés, avec celle qu'il aurait soumise s'il détenait des contrats à terme à la place d'options. Ce résultat est intuitif puisque $a$ unités d'option procurent le même paiement que zéro contrat à terme si le prix de l'action est inférieur au prix d'exercice et que $a$ contrats à terme s'il lui est supérieur. Toutefois, si l'investisseur est en position longue sur l'option, il ne passe pas d'une demande à l'autre au prix d'exercice $K:$ il sait que sa demande a un impact sur le prix d'échange et soumet une demande croissante en le prix, afin d'augmenter de façon optimale la probabilité d'exercice

FIGURE 1

Fonctions de demande pour $a>0$ et $a<0$.
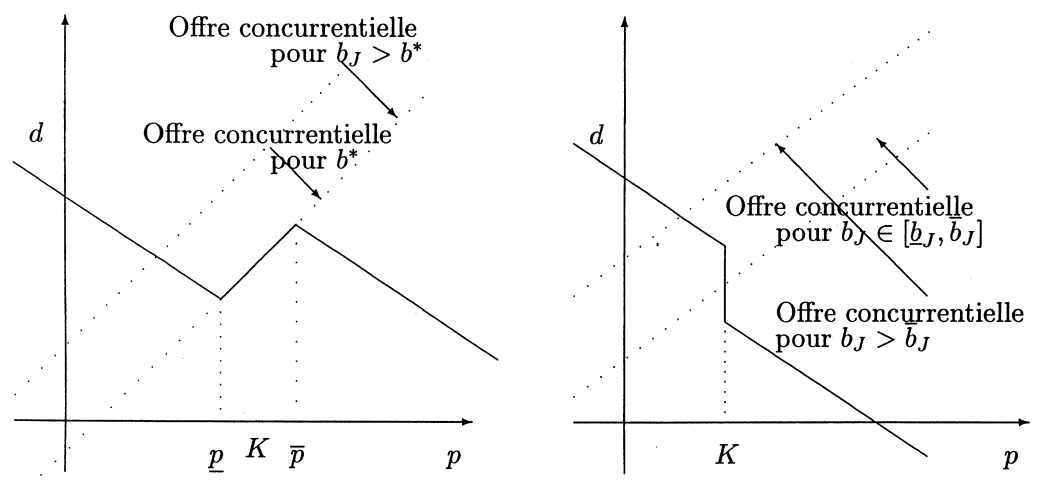
de l'option. En conséquence, le prix « saute » de $p$ à $\bar{p}$ quand l'offre de couverture $b_{J}$ atteint $b^{*}$. Plus $a>0$ est élevé, plus Te saut est important ( $p$ diminue et $\bar{p}$ augmente avec $a$ ).

Différentes courbes d'offre ont été tracées sur la figure 1 ainsi que la fonction de demande optimale de l'investisseur suivant sa position, longue ou courte. On obtient les prix d'équilibre à l'intersection des courbes d'offre et de demande. Si la position du monopole sur l'option est longue $(a>0)$, il existe un unique prix annulant la demande excédentaire, excepté quand $\tilde{b}_{J}=b^{*}$. Ce dernier cas ne se produit qu'avec une probabilité nulle et est le seul où le prix d'échange peut appartenir à $[\underline{p}, \bar{p}]$. Les prix entre $\underline{p}$ et $\bar{p}$ et en particulier $K$ ne sont donc presque sûrement jamais atteints à l'équilibre. À l'opposé, quand le monopole détient une position courte sur l'option $(a<0)$, le prix d'exercice $K$ équilibre le marché dès que la demande de couverture concurrentielle est située dans un certain intervalle.

La demande décrite dans la proposition est optimale pour toute distribution continue de $\tilde{b}_{J}$. Si celle-ci est bornée, toute autre demande générant la même distribution du prix d'échange est également optimale.

\section{5 Équilibre avec plusieurs investis- seurs stratégiques}

Si plusieurs investisseurs adoptent des comportements stratégiques, nous allons montrer que des équilibres multiples, caractérisés par des issues différentes, peuvent exister. Nous recherchons tout d'abord des équilibres, appelés équilibres linéaires, pour lesquels les demandes stratégiques sont des fonctions linéaires du prix. Pour cela, nous nous appuyons sur le cas où le contrat dérivé est un contrat à terme. Il existe alors un unique équilibre linéaire. Avec des options, des stratégies similaires peuvent former un équilibre, mais sous des conditions fortes sur les portefeuilles initiaux. Nous recherchons ensuite un équilibre non linéaire quand les dotations en option des investisseurs non concurrentiels sont identiques.

\section{1 Équilibre linéaire}

Un équilibre linéaire s'obtient facilement si les investisseurs stratégiques détiennent des contrats à terme à la place des options ${ }^{13}$. En effet, d'après la proposition 1 , une meilleure réponse à une offre résiduelle linéaire est ellemême linéaire.

Plus précisément, la demande $d_{\lambda, a_{i}, b_{i}}$ définie dans l'équation (5), de pente $-1 /\left[(\lambda+\rho) \sigma_{v}^{2}\right]$, constitue une meilleure réponse à une offre résiduelle de

13. La construction généralise celle de KYLE [1989] qui considère une offre concurrentielle inélastique en l'absence de contrats à terme. 
pente $1 /\left(\lambda \sigma_{v}^{2}\right)$. Pour obtenir un équilibre il faut que la courbe d'offre soit correctement anticipée. Puisque celle-ci est constituée des offres des $n-1$ autres investisseurs stratégiques et de l'offre compétitive, sa pente est correctement anticipée si le coefficient $\lambda$ vérifie l'équation

$$
\frac{1}{\lambda}=\frac{n-1}{\lambda+\rho}+\frac{1}{\rho_{J}} \text {. }
$$

L'existence d'un unique équilibre linéaire s'en déduit aisément.

Proposition 3 Soient $n$ investisseurs stratégiques, détenant les portefeuilles $\left(a_{i}, b_{i}\right)$ de contrat à terme et d'action pour $i=1, . ., n$ et $\lambda$ l'unique solution positive de l'équation (8).

Alors les stratégies $\left(d_{\lambda, a_{i}, b_{i}}, i=1, \ldots, n\right)$ forment un équilibre dans lequel le prix d'échange est donné par :

$$
p\left(a_{I}, b_{I}, b_{J}\right)=\bar{v}-\left(\frac{n}{\lambda+\rho}+\frac{1}{\rho_{J}}\right)^{-1}\left(\frac{\rho b_{I}-\lambda a_{I}}{(\lambda+\rho)}+b_{J}\right) \sigma_{v}^{2}
$$

Le paramètre $\lambda$ mesure la déviation par rapport à l'équilibre concurrentiel, puisque la demande $d_{0, a_{i}, b_{i}}$ est égale à la demande concurrentielle. L'hypothèse de comportements stratégiques a donc deux effets. Le premier est de réduire la sensibilité des demandes au prix, indépendamment des détentions de contrats à terme. Le second est d'accroître ou de diminuer le niveau de la demande d'un investisseur stratégique en fonction de sa détention de contrats à terme, afin d'accroître ou de diminuer le prix d'échange.

Remplaçons maintenant le contrat à terme par l'option. Si le prix d'échange, tel qu'il est donné par l'équation (9), est toujours supérieur au prix d'exercice $K$, le paiement de l'option est identique à celui du contrat à terme. Il paraît alors vraisemblable que les demandes $\left(d_{\lambda, a_{i}, b_{i}}\right)$ forment aussi un équilibre. Ce n'est vrai que sous des conditions supplémentaires. En effet, un investisseur en position longue sur l'option subit une perte moindre sur une option que sur un contrat à terme lorsque le prix d'échange est inférieur au prix d'exercice.

Suivant le même principe, on peut chercher un autre équilibre linéaire pour lequel l'option n'est jamais exercée. Il suffit de considérer l'équilibre linéaire $\left(d_{\lambda, 0, b_{i}}\right)$ obtenu lorsque les investisseurs stratégiques ne détiennent pas de contrat à terme. Si le prix d'échange est toujours inférieur au prix d'exercice $K$, le paiement de l'option est alors nul. Quand les investisseurs détiennent des options, les stratégies ci-dessus forment aussi un équilibre si aucun agent en position longue sur l'option n'a intérêt à accroître sa demande afin d'exercer celle-ci.

PROPOSITION 4 Soient $n$ investisseurs stratégiques détenant les portefeuilles $\left(a_{i}, b_{i}\right)$ d'option et d'action, pour $i=1, . ., n$ et $\lambda$ l'unique solution positive de l'équation (8). Étant donné les distributions des portefeuilles des intervenants non concurrentiels, 
1. les demandes $\left(d_{\lambda, a_{i}, b_{i}}, i=\underset{\sim}{1}, . ., n\right)$ forment un équilibre si la borne supérieure de la distribution de $\tilde{b}_{J}$ est suffisamment petite ;

2. les demandes $\left(d_{\lambda, 0, b_{i}}, i=1, \ldots, n\right)$ forment un équilibre si la borne inférieure de la distribution de $\tilde{b}_{J}$ est suffisamment grande.

Si tous les investisseurs stratégiques détiennent des positions longues sur l'option et sont en nombre supérieur à deux, il existe des distributions de l'offre de couverture pour lesquelles les deux équilibres existent simultanément.

Les bornes sur la distribution de $\tilde{b}_{J}$ propres à assurer l'existence d'un équilibre se calculent aisément ${ }^{14}$. Conformément à l'intuition, le premier équilibre, pour lequel l'option sera toujours exercée, a d'autant plus de chance d'exister que les dotations des investisseurs stratégiques sont grandes sur l'option, faibles sur l'action et que la différence $\bar{v}-K$ est grande.

La multiplicité des équilibres est liée aux complémentarités stratégiques induites par la détention de l'option (positions longues). Plus la demande d'un investisseur est élevée, moins il est coûteux pour les autres d'accroître leurs demandes afin que le prix augmente au-delà du prix d'exercice. En conséquence, si un investisseur anticipe que tous les autres aient choisi la demande « élevée » $d_{\lambda, a, b}$, il peut être optimal pour lui de choisir également cette demande, ce qui conduit au premier équilibre. Mais si tous les autres ont choisi la demande « faible » $d_{\lambda, 0, b}$, il préférera sa demande faible lorsque les possibilités d'exercice sont suffisamment amoindries.

Bien sûr, l'importance de ces complémentarités stratégiques dépend des opportunités de manipulation, elles-mêmes fonctions de l'aversion pour le risque. Plus un investisseur présente de l'aversion pour le risque ( $\rho$ élevé), moins il réagit face à une courbe d'offre donnée : sa fonction de meilleure réponse est moins pentue. À l'équilibre, le coefficient $\lambda$ - qui mesure l'impact de la concurrence imparfaite - croît avec le coefficient d'aversion pour le risque $\rho$. Il en est de même de l'écart entre les demandes associées aux deux équilibres $\left(d_{\lambda, a_{i}, b_{i}}\right.$ et $\left.d_{\lambda, 0, b_{i}}\right)$, comme de celui entre les prix correspondants. En conséquence, les deux équilibres ont plus de chances d'exister (simultanément ou non) quand l'aversion pour le risque des investisseurs est grande. À l'opposé, si les investisseurs stratégiques sont presque neutres vis-à-vis du risque, l'impact de la concurrence imparfaite est faible : pour les prix différant de $\bar{v}$, les investisseurs soumettent des demandes très importantes à l'achat ou à la vente si bien que le prix d'échange est proche de $\bar{v}$, et la manipulation trop coûteuse ; de ce fait les détentions d'option ne jouent plus de rôle ${ }^{15}$.

14. Par exemple, si tous les investisseurs stratégiques détiennent les mêmes portefeuilles $(a, b)$, le premier équilibre existe si $b_{J}$ est toujours inférieur à $\bar{b}$, et le second si $b_{J}$ est toujours supérieur à $\underline{b}$ avec

$$
\bar{b}=\frac{1}{\lambda+\rho}\left[\frac{2 \lambda+\rho}{\lambda \sigma_{v}^{2}}(\bar{v}-K)-n \rho b+\lambda(n-1 / 2) a\right] \text { et } \underline{b}=\bar{b}-\frac{\lambda}{\lambda+\rho}(n-1) a .
$$

Si $a>0$ et $n \geqslant 2$, alors $\underline{b}<\bar{b}$ : les deux équilibres existent simultanément si le support de la distribution de $b_{J}$ est inclus dans l'intervalle $[\underline{b} ; \bar{b}]$.

15. Ce raisonnement ne subsiste plus dans le cas du monopole puisqu'il fait face à une offre exogène et gagne ainsi à manipuler le prix même s'il est neutre vis-à-vis du risque (il suffit de prendre $\rho=0$ dans les propositions 1 et 2). 
Ainsi, contrairement au cas du contrat à terme pour lequel un équilibre linéaire existe et est unique, la convexité du paiement de l'option peut entraîner l'inexistence d'un équilibre linéaire ou au contraire induire leur multiplicité. En particulier, dans le cas vraisemblable où la valeur fondamentale espérée $\bar{v}$ est proche du prix d'exercice $K$ et l'offre de couverture suffisamment variable, aucun des équilibres linéaires ci-dessus n'existe. Nous étudions maintenant un équilibre non linéaire.

\section{2 Équilibre dans le cas symétrique}

La recherche d'un équilibre présente deux difficultés : d'une part l'espace des stratégies constitue un continuum, de l'autre la convexité du paiement de l'option induit des discontinuités dans la fonction de meilleure réponse (sauf si l'exercice de l'option est dégénéré comme dans les cas considérés cidessus). De ce fait l'offre résiduelle sera elle-même discontinue. Afin de simplifier le problème, nous supposons ici les portefeuilles des investisseurs non concurrentiels identiques, avec des positions longues sur l'option. Cette hypothèse est raisonnable dans la mesure où les comportements stratégiques sont le fait de grosses institutions financières à même d'adopter des positions similaires. Nous recherchons alors un équilibre symétrique, pour lequel les stratégies des investisseurs sont identiques. Des demandes linéaires par morceaux peuvent, sous certaines conditions, former un équilibre symétrique, comme l'énonce la proposition suivante.

Proposition 5 Soient $n$ investisseurs stratégiques détenant tous le même portefeuille $(a, b)=\left(a_{I} / n, b_{I} / n\right)$, avec $a>0$, et $\lambda$ l'unique solution positive de l'équation (8). Supposons de plus

$$
0 \leqslant \frac{\bar{v}-K}{\rho \sigma_{v}^{2}}-b \leqslant a
$$

et la distribution de $\tilde{b}_{J}$ continue. Alors il existe un équilibre symétrique dans lequel la demande est continue, linéaire par morceaux avec deux points de rupture $\underline{p}_{n}<K<\bar{p}_{n}$ et donnée par :

$$
d_{\lambda, 0, b}(p) \text { pour } p \leqslant \underline{p}_{n} \text {, et } d_{\lambda, a, b}(p) \text { pour } p \geqslant \bar{p}_{n} .
$$

Les prix d'équilibre correspondent respectivement à $p\left(0, n b, b_{J}\right)$ pour $p<\underline{p}_{n}$ et $p\left(n a, n b, b_{J}\right)$ pour $p>\bar{p}_{n}$, la fonction $p($.$) étant définie par$ l'équation (9) dans la proposition 3.

La condition (10) a de grandes chances d'être satisfaite si les positions sur l'option d'un investisseur stratégique sont suffisamment larges. La première inégalité implique que sa demande concurrentielle au prix $K$ est positive. En l'absence d'option, l'investisseur stratégique souhaiterait alors décroître légèrement sa demande, tout en la maintenant positive, afin d'obtenir un prix plus 
faible. Toutefois, s'il détient suffisamment d'option (comme requis par l'inégalité de droite), il préfère augmenter sa demande, quitte à subir une perte sur l'action, afin d'augmenter le prix d'échange et d'exercer l'option. Sous la condition (10), la détention d'option est donc le motif prépondérant des manipulations.

Finissons par deux remarques. Premièrement, comme dans le cas du monopole, les fonctions de demande sont localement croissantes. Ceci justifie l'utilisation des ordres à seuil de déclenchement. Deuxièmement, la condition (10) et les conditions d'existence des deux équilibres linéaires peuvent être simultanément vérifiées. Dans ce cas, nous pouvons exhiber trois équilibres correspondant à des distributions de prix d'équilibre différentes. Cette multiplicité d'équilibre résulte des complémentarités stratégiques induites par les détentions positives d'option.

Nous avons étudié d'autres situations simples, par exemple les investisseurs stratégiques sauf un ne détiennent pas d'option. Dans ce cas, il existe au plus un équilibre linéaire (par défaut de complémentarités stratégiques), qui de plus n'existe que sous des conditions fortes (qui assurent que l'option est soit jamais soit toujours exercée). En général, comme dans le cas du monopole étudié en partie 4, la demande de l'unique détenteur d'option ne sera pas linéaire, si bien que la meilleure réponse des autres investisseurs, bien qu'ils ne détiennent pas d'option, sera elle aussi non linéaire. Comme ci-dessus, nous ne trouvons pas d'équilibres linéaires par morceaux sans condition supplémentaire.

\section{Conclusion}

Nous avons montré dans cet article que le comportement non concurrentiel de détenteurs d'option pouvait avoir, à sa date d'exercice, un impact important sur le prix de l'action sous-jacente. Plusieurs implications empiriques quant au comportement des investisseurs et au processus de formation des prix découlent de nos résultats. Tout d'abord, il peut être optimal pour un investisseur de soumettre une fonction de demande localement croissante par rapport au prix. Cela permet de rationaliser l'usage des ordres stop ou à seuil de déclenchement ${ }^{16}$ lors d'une procédure de fixing. Par ailleurs, la convexité du paiement de l'option génère des externalités positives entre les détenteurs (au sens strict) de l'option qui, à leur tour, génèrent une multiplicité d'équilibre. Nous avons notamment montré que trois équilibres, avec des supports de prix différents, peuvent simultanément exister dès que le nombre de manipulateurs potentiels est supérieur à deux. Cette multiplicité d'équilibre peut perturber la coordination entre les investisseurs, ce qui laisse supposer un effet déstabilisant de la liquidation de l'option sur le marché de l'action sousjacente. Evidemment une étude empirique serait souhaitable, même si de

16. Ordres d'achat (respectivement de vente) qui ne sont exécutés que si le prix augmente (respectivement diminue) suffisamment. 
l'avis des professionnels eux-mêmes, des manipulations ont effectivement lieu.

Une manipulation du prix n'est bien sur profitable que si le prix d'échange dépend du flux des demandes. Dans notre modèle, cette dépendance provient de l'aversion pour le risque des investisseurs. Des résultats similaires pourraient être obtenus sous d'autres hypothèses, dans d'autres structures de marché. Par exemple, dans un marché neutre au risque avec teneurs de marché, le prix dépend du flux dès qu'il y a des asymétries d'information.

Finalement, notre étude porte sur le marché de l'action sous-jacente à une date bien précise : la liquidation de l'option. Il serait intéressant d'étendre notre analyse à des périodes antérieures, quand option et action sont simultanément échangées.

\section{Preuves}

Nous utiliserons la fonction $\pi$ qui correspond à un « profit » corrigé du risque si l'investisseur détient $a$ unités de contrat à terme :

$$
\pi_{a}(p, x)=x(\bar{v}-p)+a(p-K)-\frac{\rho}{2}(x+b)^{2} \sigma_{v}^{2} .
$$

PREUVE DE LA PROPOSITION 1. Soit un monopole détenant $a$ contrats à terme et $b$ unités d'action. Il fait face à la fonction d'offre aléatoire $o_{\gamma}(p)+\tilde{\varepsilon}$ $=\frac{p-\bar{v}}{\gamma \sigma_{v}^{2}}+\tilde{\varepsilon}$. Supposons tout d'abord qu'il observe la réalisation $\varepsilon$ de $\tilde{\varepsilon}$. Il choisit alors sa transaction préférée $(p, x)$ sur la courbe d'offre de façon à maximiser $-E[\exp \{-\rho(x(\tilde{v}-p)+b \tilde{v}+a(p-K))\}]$. Comme il connaît $\varepsilon$, la seule source d'incertitude est $\tilde{v}$, qui est normalement distribuée ; par des arguments standards, le problème équivaut à :

$$
\operatorname{Max}_{(p, x)} \pi_{a}(p, x) \text { sous la contrainte } x=\frac{p-\bar{v}}{\gamma \sigma_{v}^{2}}+\varepsilon \text {. }
$$

Du fait de la concavité de la fonction à maximiser, il existe une unique solution qui vérifie à la fois les contraintes du premier et deuxième ordre :

$$
\frac{\partial p}{\partial x}(a-x)+(\bar{v}-p)+a(p-K)-\rho(x+b) \sigma_{v}^{2}=0, \text { avec } \frac{\partial p}{\partial x}=\gamma \sigma_{v}^{2}
$$

L'équation (12) est exactement celle de la demande $d_{\gamma, a, b}$ donnée par (5). La solution du problème est donc unique, donnée par le point d'intersection entre la demande $d_{\gamma, a, b}$ et la courbe d'offre que l'on notera $\left(p_{a}(\varepsilon), x_{a}(\varepsilon)\right)$ (il dépend également de $\gamma$ et $b$ ). On en déduit immédiatement que la fonc- 
tion $d_{\gamma, a, b}$ permet à l'investisseur d'obtenir l'échange qu'il préfère, même s'il n'observe pas $\varepsilon$. Des calculs simples aboutissent à :

$$
p_{a}(\varepsilon)=\bar{v}-\left(\frac{1}{\gamma+\rho}+\frac{1}{\gamma}\right)^{-1}\left(\frac{\rho b-\gamma a}{\gamma+\rho}+\varepsilon\right) \sigma_{v}^{2},
$$

soit l'équation (6).

Notons $\pi_{a}(\varepsilon)$ la valeur du programme (11). Le lemme 1 regroupe des résultats utiles pour la suite.

LEMME 1 Soit un investisseur détenant le portefeuille $(a, b)$ de contrats à terme et d'action. Quand $\varepsilon$ crôt :

1. Le profit $\pi_{a}$ est décroissant puis croissant, avec le minimum obtenu pour l'échange correspondant au prix $\left(\bar{v}-\rho \sigma_{v}^{2}(b+a)\right.$ et à la demande $a$.

2. La différence $\pi_{a}-\pi_{0}$ crô̂t (respectivement décroît) si $a>0$ (resp. $a<0)$; elle est négative pour tout $\varepsilon$ tel que $p_{a}(\varepsilon)=K$ et positive pour tout $\varepsilon$ tel que $p_{0}(\varepsilon)=K$.

\section{PREUVE DU LEMME 1.}

1. Par définition $\pi_{a}(\varepsilon)$ est la valeur du programme (11). D'après le théorème de l'enveloppe, sa dérivée est donnée par :

$$
\pi_{a}^{\prime}(\varepsilon)=(\bar{v}-p)-\rho(x+b) \sigma_{v}^{2}
$$

calculée sur la solution $\left(p_{a}(\varepsilon), x_{a}(\varepsilon)\right)$. Comme cette solution vérifie (12), i.e. appartient à la fonction de demande $d_{\gamma, a, b}$, on peut aussi écrire :

$$
\pi_{a}^{\prime}(\varepsilon)=a-x_{a}(\varepsilon)=a-d_{\gamma, a, b}\left(p_{a}(\varepsilon)\right) .
$$

Quand $\varepsilon$ croît, à partir de $-\infty$, le prix $p_{a}(\varepsilon)$ décroît de $+\infty$ à $-\infty$, d'après (6). La transaction préférée parcourt le fonction de demande $d_{\gamma, a, b}$ du SE au NW. En conséquence, le profit décroît puis croît à nouveau. Le minimum est atteint quand $d_{\gamma, a, b}\left(p_{a}(\varepsilon)\right)=a$, soit pour le $\operatorname{prix} p_{a}(\varepsilon)=\bar{v}-\rho \sigma_{v}^{2}(b+a)$.

2. D’après (13),

$$
\pi_{a}^{\prime}(\varepsilon)-\pi_{0}^{\prime}(\varepsilon)=-\left(p_{a}(\varepsilon)-p_{0}(\varepsilon)\right)-\rho\left(x_{a}(\varepsilon)-x_{0}(\varepsilon)\right) \sigma_{v}^{2} .
$$

Comme les deux points appartiennent à la courbe d'offre associée à $\varepsilon$, on obtient : $\pi_{a}^{\prime}(\varepsilon)-\pi_{0}^{\prime}(\varepsilon)=\left(p_{a}(\varepsilon)-p_{0}(\varepsilon)\right)(1+\rho / \gamma)$. D'après $(6)$, cette expression est du même signe que $a$, d'où le résultat.

Finalement, si $p_{a}(\varepsilon)=K$, le paiement du contrat à terme est nul, si bien que le profit sans contrat à terme est nécessairement supérieur ou égal à celui avec $a$ contrats à terme. La preuve est similaire dans le cas où $p_{0}(\varepsilon)=K$. 
PREUVE DE LA PROPOSITION 2. Le monopole fait face à la courbe d'offre linéaire paramétrée par $\rho_{J}$, donnée dans l'équation (7). Afin de simplifier les notations, nous écrivons désormais : $d_{a}=d_{\rho_{J}, a, b}$ et $d_{0}=d_{\rho_{J}, 0, b}$. Considérons la courbe d'offre associée à $b_{J}$. Pour les prix $p$ inférieurs à $K$, le profit le long de la courbe d'offre correspond au profit en l'absence de contrat à terme et pour les prix supérieurs à $K$ à celui avec $a$ contrats à terme.

1) Nous examinons en premier lieu le cas d'une détention positive d'option : $a>0$. Le profit est concave en le prix sur chacun des intervalles $]-\infty, K]$ et $[K,+\infty$ [ mais pas globalement car sa dérivée présente un saut vers le haut en $p=K$ du fait de $a$. Il faut donc comparer les maxima du profit obtenus sur chacun des deux intervalles. Plus précisément, d'après le lemme 1 , ils sont obtenus

- en $\operatorname{Max}\left(p_{0}\left(b_{J}\right), K\right)$ pour $p \leqslant K$

- et en $\operatorname{Max}\left(p_{a}\left(b_{J}\right), K\right)$ pour $p \geqslant K$.

Notons que $p_{0}\left(b_{J}\right)<p_{a}\left(b_{J}\right)$. Le cas où l'un des deux extrema locaux est $K$ ne présente pas de difficultés. Par continuité, le maximum global correspond à l'autre extremum. Par exemple, si $p_{0}\left(b_{J}\right)>K$, alors $p_{a}\left(b_{J}\right)$ est un maximum global. Il reste à étudier la cas $p_{0}\left(b_{J}\right)<K<p_{a}\left(b_{J}\right)$. D'après le lemme 1 , il existe une valeur de rupture $b^{*}$ pour laquelle l'investisseur est indifférent entre les deux prix $p=p_{0}\left(b^{*}\right)$ et $\bar{p}=p_{a}\left(b^{*}\right)$; il préfère le prix $p_{0}\left(b_{J}\right)$ pour les valeurs de $b_{J}$ supérieures à $b^{*}$ et il préfère le prix $p_{a}\left(b_{J}\right)$ pour les valeurs inférieures. De plus, $p<K<\bar{p}$.

La fonction linéaire par morceaux $d$ définie dans la proposition 2 coïncide avec $d_{0}$ pour $p<p$, avec $d_{a}$ pour $p>\bar{p}$ et avec la courbe d'offre associée à $b^{*}, d(p)=o_{J}(p)+b^{*}$, pour $\underline{p} \leqslant p \leqslant \bar{p}$. Elle est continue puisque par construction, les deux points extrêmes $\left(d_{0}(p), p\right)$ et $\left(d_{a}(\bar{p}), \bar{p}\right)$ appartiennent tous deux à la courbe d'offre associée à $b^{*}$. La demande $d$ permet à l'investisseur d'obtenir sa transaction préférée sur la courbe d'offre sans observer $b_{J}$, pour tout $b_{J} \neq b^{*}$. En effet, pour chaque réalisation $b_{J}$, le prix d'échange $p$ vérifie $d(p)-o_{J}(p)=b_{J}$. Le membre de gauche est strictement décroissant sauf sur l'intervalle $[\underline{p}, \bar{p}]$ où il est constant, égal à $b^{*}$. En conséquence, pour $b_{J}<b^{*}$, le prix d'échange est unique et égal à $p_{a}\left(b_{J}\right)$. De même, si $b_{J}>b^{*}$, le prix d'échange est donné par $p_{0}\left(b_{J}\right)$. Donc, si $b^{*}$ est un point négligeable de la distribution de $\tilde{b}_{J}$, la demande $d$ constitue une meilleure réponse face à la courbe d'offre.

2) Supposons maintenant $a<0$. Le profit le long de la courbe d'offre est concave en $p$ puisque maintenant sa dérivée présente un saut vers le bas en $p=K$. L'inégalité $p_{0}\left(b_{J}\right)>p_{a}\left(b_{J}\right)$ est toujours vérifiée. Étant donné $b_{J}$, la transaction préférée est obtenue

- soit en un prix satisfaisant la condition du premier ordre, c'est-à-dire $p_{0}\left(b_{J}\right)$ quand ce prix est inférieur à $K$ et $p_{a}\left(b_{J}\right)$ quand ce prix est supérieur à $K$, 
- soit en $K$ quand $p_{0}\left(b_{J}\right)>K>p_{a}\left(b_{J}\right)$.

Ce dernier cas se produit dès que la réalisation $b_{J}$ appartient à un intervalle donné, noté $\left[\underline{b}_{J}, b_{J}\right]$. La demande figurant dans la proposition 2 permet à l'investisseur d'obtenir la même transaction.

PREUVE DE LA PROPOSITION 3. Considérons un investisseur stratégique $i$ et supposons que tous les autres investisseurs stratégiques $k \neq i$ soumettent la fonction de demande linéaire $d_{\lambda, a_{k}, b_{k}}$. L'agent $i$ fait alors face à une courbe d'offre résiduelle linéaire de pente $\frac{1}{\gamma \sigma_{v}^{2}}$, avec $\frac{1}{\gamma}=\frac{n-1}{\lambda+\rho}+\frac{1}{\rho_{J}}$. D'après la proposition 1, la meilleure réponse de l'agent $i$ est donnée par $d_{\gamma, a_{i}, b_{i}}$. Un équilibre avec contrats à terme est donc obtenu si $\gamma=\lambda$, i.e. si $\lambda$ est solution de l'équation (8).

PREUVE DE LA PROPOSITION 4. Nous recherchons des conditions sous lesquelles les stratégies $\left(d_{\lambda, a_{i}, b_{i}}, i \in I\right)$ forment un équilibre avec option, quand $\lambda$ est l'unique solution de l'équation (8).

Supposons que tous les investisseurs stratégiques $k$, pour $k$ différent de $i$, soumettent la demande $d_{\lambda, a_{k}, b_{k}}$. Alors $i$ fait face à la courbe d'offre résiduelle $o_{\lambda}(p)+\tilde{\eta}$ avec $\tilde{\eta}=\tilde{b}_{J}-(n-1) \sum_{k \neq i} \frac{\lambda a_{k}-\rho b_{k}}{(\lambda+\rho)}$. En utilisant un argument similaire à celui de la proposition 2, la meilleure réponse de $i$ est $d_{\lambda, a_{i}, b_{i}}$ si le prix d'échange est plus grand que $K$ et s'il n'a pas intérêt à dévier en soumettant la demande $d_{\lambda, 0, b_{i}}$. Des calculs simples mais fastidieux permettent de montrer que c'est le cas si :

$$
\frac{\bar{v}-K}{\sigma_{v}^{2}}+\frac{\lambda}{2 \lambda+\rho}\left\{\lambda a_{I}-\lambda a_{i} / 2-\rho b_{I}-(\lambda+\rho) \tilde{b}_{J}\right\}>0 .
$$

La condition (14) doit être vraie pour tout $i$ presque sûrement. On obtient ainsi une borne supérieure sur la distribution de $\tilde{b}_{J}$. De même, les stratégies $\left(d_{\lambda, 0, b_{i}}, i \in I\right)$ forment un équilibre dans lequel l'option n'est jamais exercée si la condition suivante est vérifiée avec probabilité un :

$$
\frac{\bar{v}-K}{\sigma_{v}^{2}}+\frac{\lambda}{2 \lambda+\rho}\left\{\lambda a_{i} / 2-\rho b_{I}-(\lambda+\rho) \tilde{b}_{J}\right\}<0 .
$$

La condition (15) est satisfaite quand toutes les réalisations possibles de $\tilde{b}_{J}$ sont suffisamment élevées. Si les investisseurs stratégiques détiennent tous des montants positifs d'option, $\left(a_{i} \geqslant 0, \forall i\right)$, il existe des distributions bornées de $\tilde{b}_{J}$ telles que les conditions (14) et (15) soient simultanément vérifiées ; dans ce cas, les deux équilibres co-existent.

PRINCIPE DE LA PREUVE DE LA PROPOSITION 5. La preuve complète est disponible auprès des auteurs. Pour rechercher l'équilibre, nous appliquons la 
même méthode que pour le monopole. Cependant, un investisseur stratégique ne fait plus face à une offre résiduelle linéaire mais linéaire par morceaux (et ce, même si l'offre de couverture $b_{J}$ est observable), d'où une difficulté supplémentaire. La courbe d'offre résiduelle est en effet moins pentue pour les prix proches du prix d'exercice $K$ (entre $\underline{p}_{n}$ et $\bar{p}_{n}$ ) si les autres investisseurs soumettent effectivement les demandes d'équilibre. Dans cette zone de prix, la manipulation est donc moins coûteuse. Sous la condition (10), aucun des prix correspondants ne sont atteints à l'équilibre. Comme dans le cas du monopole, il est facile de montrer qu'une demande contingente aux prix permet d'obtenir dans toutes circonstances la même issue qu'une demande conditionnée sur $b_{J}$ dans le cas où celui-ci est observable.

Plus précisément, la preuve est composée de quatre étapes.

La première consiste en la construction de l'unique demande $d$ vérifiant les propriétés suivantes : $d$ est continue; il existe des valeurs $\underline{p}$ et $\bar{p}$, avec $p<\bar{p}$, telles que $d$ coïncide avec $d_{0}$ pour $p$ inférieur à $p$ et avec $d_{a}$ pour $p$ supérieur à $\bar{p}$; la pente de $d$ sur l'intervalle $[p, \bar{p}]$ est telle que la demande agrégée soit plate sur cette intervalle, pour peu que tous les investisseurs stratégiques soumettent $d ; p<K<\bar{p}$ et $\pi\left(p, d_{0}(p)\right)=\pi\left(\bar{p}, d_{a}(\bar{p})\right)$.

Dans une seconde étape, nous considérons le profit d'un investisseur stratégique qui pense que tous les autres investisseurs non concurrentiels soumettent la demande $d$. Il anticipe l'offre $o(p)+b_{J}$, avec

$$
o(p)=o_{J}(p)-(n-1) d(p) .
$$

Sur chaque intervalle délimité par $p, K$, et $\bar{p}$, son profit est concave mais la dérivée présente des discontinuités en chaque extrémité, dont le sens dépend non seulement de $a$ mais aussi de la transaction associée. A priori, on devrait comparer les maxima locaux sur chacun des quatre intervalles. En utilisant la condition (10), une troisième étape met en évidence deux maxima pour $p \leqslant K$ et $p \geqslant K$ qui sont respectivement atteints au point d'intersection avec les demandes $d_{0}$ et $d_{a}$. La détermination du maximum global parmi les deux extrema locaux résulte de la quatrième étape. 


\section{- Références}

[1] BACK K. (1993). - « Asymmetric Information and Options », The Review of Financial Studies, 6, pp. 435-472.

[2] Bhattacharya U. and M. Spiegel (1991). - « Insiders, Outsiders, and Market Breakdowns », The Review of Financial Studies, 4, pp. 255-282.

[3] BikHChandani S. and C. F. Huang (1993). - « The economics of treasury securities markets », Journal of Economic Perspectives, 7, pp. 117-134.

[4] Danthine J.-P. (1978). - «Information, futures prices and stabilizing speculation », Journal of Economic Theory, 17, pp. 79-98.

[5] Demange G., and G. Laroque (1999). - « Efficiency and options on the market index », Economic Theory, 14, pp. 227-235.

[6] Genotte G. and Leland H. (1990). - « Hedging and Crashes », American Economic Review, 80, pp. 999-1021.

[7] JARROW R.A. (1992). - « Market manipulation, bubbles, corners and short squeezes », Journal of Financial and quantitative Analysis, 27, pp. 311-336

[8] JegadeEsh N. (1993). - « Treasury Auction and the Salomon Squeeze », Journal of Finance, 48, pp. 1403-1419.

[9] Klemperer P. D. and M. A. Meyer (1989). - « Supply function equilibria in oligopoly under uncertainty », Econometrica, 57, pp. 1243-1278.

[10] Kumar P. and SEPPI D. (1992). - « Futures manipulation with "cash settlement", Journal of Finance, 47, pp. 1485-1502.

[11] KyLE A. (1989). - «Informed speculation with imperfect competition », Review of Economic Studies, 56, pp. 317-356.

[12] STEIN J. (1987). - « Informational externalities and welfare-reducing speculation », Journal of political economy, 95, pp. 1123-1145.

[13] Ross S. (1976). - « Options and Efficiency », Quarterly Journal of Economics, 90, pp. 75-89.

[14] TuRnovsky S. (1983). - « The determination of spot and futures prices with storable commodities », Econometrica, 51, pp. 1362-1387.

[15] Wilson R. B. (1979). - « Auctions of shares », Quarterly Journal of Economics, 93, pp. 675-89. 